présentation claire et précise de ce que sont les coûts de transaction. Combien de fois avons-nous pu lire des références aux travaux classiques de Coase et de Williamson tout en demeurant sur notre faim? Ici tout est clair comme l'eau de source. On y trouve vers la fin une belle citation de Henry Ford : «Diminuer les salaires ne diminue pas les coûts. Cela les augmente. La seule façon d'obtenir un produit à faible coût est de payer cher un service humain de haut niveau ».

Revenons au développement durable avec P. Bello qui en fait l'analyse au niveau de l'entreprise à partir des critères suivants : l'action sociale, la gestion environnementale, le gouvernement d'entreprise, les relations contractuelles externes de l'entreprise, les relations avec la société civile, sportive et culturelle, etc. Ces critères peuvent varier légèrement selon la taille des entreprises, leur statut et la nature de leurs activités.

En relation avec l'évaluation multicritères, $\mathrm{N}$. Sassenou à son tour évoque la responsabilité sociale de l'entreprise en l'associant aux considérations suivantes : la rentabilité économique, le respect de l'environnement, l'implication dans son milieu de concert avec la société civile, l'amélioration de la qualité des produits et la satisfaction des clients, l'adoption d'une GRH avec vision dans le long terme, la mise en pratique des principes du gouvernement d'entreprise (communication, transparence dans relations avec les actionnaires...).

Ce dernier concept n'est pas courant. L'auteur juge bon de préciser qu'il correspond à l'ensemble des dispositifs permettant d'harmoniser le comportement des dirigeants avec l'intérêt des actionnaires. Ces derniers ne sont pas toujours les porte-étendards de tout ce qui relève de l'éthique, mais comme il y a des exceptions à tout, l'auteur a jugé bon de présenter le Domini Social Index : un indice socialement responsable basé sur les titres américains cotés. Sont exclues de cet indice les entreprises dont le chiffre d'affaires en armement dépasse les $2 \%$, celles qui œuvrent dans le secteur du tabac, de l'alcool (dommage pour la Nappa Valley...) et les jeux du hasard (dommage pour nos casinos...), celles associés à l'énergie nucléaire et... celles qui faisaient affaires avec l'Afrique du Sud (c'était avant 1993...).
Comment, pour conclure, ne pas être d'accord avec les auteurs quand, dans leur conclusion, ils écrivent que le développement durable souffre de deux écueils majeurs qui se nourrissent et s'entretiennent dans une zone souvent qualifiée en termes d'amateurisme ou de dogmatisme. Ceci étant, ils ont bon espoir que d'ici peu, il sera impossible de concevoir un produit, un investissement, un barrage, un hôpital ou tout autre immeuble sans intégrer les éléments d'évaluation du développement durable dans sa triple dimension économique, sociale et environnementale. Espérons-le nous aussi.

Un ouvrage pour ceux qui s'intéressent avant tout à l'entreprise, laquelle ne peut durablement demeurer indifférente aux exigences du développement durable.

\section{Azoulay, Hervé Vive l'entreprise solidaire Paris, Eyrolles Société, 276 p.}

Même si le titre ne le laisse pas entrevoir, cet ouvrage a beaucoup en commun, du moins sur le fond, avec le précédent. Effectivement, il est également beaucoup question dans ce volume de développement durable et de la responsabilisation sociale de l'entreprise. On y trouve aussi, ici et là, de belles citations d'Henry Ford. Mais l'analogie s'arrête là car, de par sa facture et le style que favorise l'auteur, le lecteur se voit offrir une lecture d'un type très différent. Hervé Azoulay, président du Réseau associatif 95 et directeur délégué dans le groupe Schneider Electric, tire profit de sa vaste expérience pour présenter, en dix chapitres répartis à l'intérieur de trois sections, quels sont les défis que doit surmonter l'entreprise à l'aube $\mathrm{du} \mathrm{XX}^{\mathrm{e}}$ siècle.

Pour le lecteur d'Organisations et territoires, c'est sans conteste la troisième partie qui est susceptible de retenir son attention et qui pourrait éventuellement produire les effets que cherche à susciter l'auteur. Par une allusion à la mise en commun des compétences, il est question de réseau à géométrie variable. L'intelligence du réseau se manifeste en privilégiant l'autonomie, l'interdépendance, la flexibilité, la rapidité, etc. À l'intérieur de leur réseau, les organisations fonctionnent sur le mode du désordre créatif. L'atten- 
tion se trouve placée sur le global et l'orientation favorise la satisfaction des marchés sociaux. C'est encore le réseau, souligne l'auteur, qui facilite le partage des connaissances tout en coordonnant les actions dans le sens désirable. Il y voit ici une nouvelle organisation en émergence fondée sur le potentiel humain de l'entreprise, du secteur associatif et autres partenaires en se faisant le support de l'intelligence.

Dans une sous-section intitulée Les réseaux de partenaires: source d'efficacité, on peut lire un passage dont la pertinence est indéniable, à savoir que seul le travail en réseau permet d'optimiser les actions et de renforcer les expériences des organismes reliés à l'économie solidaire. Sinon, leurs intervenants continueront de présenter l'image d'acteurs œuvrant de façon dispersée plutôt qu'à l'intérieur d'un mouvement dûment coordonné. Dans la première partie, l'économie solidaire se voit définie comme l'ensemble des activités de production, de distribution et de consommation contribuant à la démocratisation de l'économie tant au niveau local que global. L'auteur revient sur ce concept susceptible de donner lieu à « un autre monde possible » pour employer le langage des altermondistes, en concevant une forme de partenariat régional appelé à servir de moteur pour le développement. Le modèle en réseau, permettant de décentraliser l'intelligence au niveau du terrain, conduit Azoulay à considérer que les acteurs économiques régionaux bénéficient de plus en plus de manœuvres pour mettre en branle leurs stratégies. Il y voit un atout considérable pour le développement d'une région. L'université, en tant qu'agent bien enraciné dans son terroir, s'avère un des acteurs clés du réseau régional. Rien de très nouveau, pourra-t-on dire! Convenons-en en signalant par contre l'originalité de la proposition d'un nouveau type de partenariat entre les associations (nos OSBL) et l'entreprise privée. Oui, l'Alcan et Centraide peuvent très bien œuvrer ensemble afin de rencontrer des objectifs sociétaux jugés fondamentaux par ce que l'auteur désigne cons- tamment par l'expression stakeholders. Suggéronslui : parties prenantes ou forces vives d'un milieu donné.

La première partie, La crise et les réformes: l'entreprise en première ligne, se rapporte davantage à la société française. Décidément, beaucoup de choses sont à changer aux pays des Jacobins. On reconnaîtra ici le fameux mal français où, en dehors d'un État très centralisateur, il n'y a point de salut. L'auteur n'hésite pas à recourir à des formules assassines pour stigmatiser le système de l'éducation française et l'élitisme qu'il favorise en donnant lieu à une société parfaitement sclérosée. S'il ne faut pas tout réformer, il faut réformer beaucoup, selon celui qui étale ses convictions en les accompagnant de multiples exemples montrant que cet autre monde possible est effectivement une réalité à notre portée et non pas une utopie. C'est pour lui la condition sine qua non de l'avènement d'un développement durable assorti de la responsabilité sociale des entreprises.

Un livre à lire autant pour sa forme que pour son contenu. L'auteur prêche par l'exemple en s'en prenant au snobisme d'une certaine élite parisienne. Il n'a pas fait son livre à partir d'autres livres. Moins de dix titres font partie de sa bibliographie et l'on ne trouve guère plus de références éparpillées ici et là. $\mathrm{Si}$ on lui sait gré de ne pas imiter ceux qu'il critique pour être du genre : «As-tu vu tout ce que j'ai lu? », on peut quand même lui reprocher gentiment de ne pas appuyer ses donnée sur des sources bien identifiées. Mais qui conteste que tout ne va pas comme dans le meilleur des mondes et que des réformes s'imposent ? Pas besoin de citer Pierre, Jean et Jacques pour formuler ce constat. L'argumentaire de l'auteur s'impose de lui-même.

\author{
André Joyal \\ Université du Québec à Trois-Rivières
}

